\title{
Whey peptides improve wound healing following caesarean section in rats
}

\author{
Junbo Wang $\dagger$, Ming Zhao $\dagger$, Rui Liang, Zhaofeng Zhang, Haifeng Zhao, Jiali Zhang, Tian Li and Yong Li* \\ Department of Nutrition and Food Hygiene, School of Public Health, Peking University Health Science Center, Beijing 100191, \\ People's Republic of China
}

(Received 3 December 2009 - Revised 23 April 2010 - Accepted 2 June 2010 - First published online 9 August 2010)

We sought to determine whether postcaesarean treatment with whey peptides (WP) affects the healing of skin and uterine incision in rats during puerperium. Forty-eight rats were randomly divided into two groups. After a caesarean section, twenty-four rats were intragastrically administered WP, while others were administered a control vehicle. On days 7, 14 and 21 after delivery, the serum total protein/albumin concentration, skin tensile strength, uterine bursting pressure, skin/uterine hydroxyproline (Hyp) concentration and histological characteristics of the scar were measured in eight rats in each group. In the WP group, the albumin concentration, skin tensile strength, uterine bursting pressure and Hyp concentration in the skin increased significantly on days 21, 7, 14 and 21, respectively. Low neutrophil count and smaller scar width in the skin incision were found in the WP group on day 7. Postcaesarean treatment with WP promoted significant wound healing in the skin incision, and had a significant wound healing potential in the uterus.

Abdominal skin: Caesarean section: Rat uterus: Whey peptides: Wound healing

Caesarean deliveries have increased remarkably over the past two decades ${ }^{(1)}$. As is known, caesarean section may cause an impaired abdominal wound. In addition, past history of caesarean section is correlated with the risk of uterine rupture during pregnancy. The consequences of rupture can be catastrophic for the mother and the baby. Women with uterine rupture have more episodes of postpartum haemorrhage (50.0 v. $0.4 \%, P<0.01)$, receive more packed cell transfusions $(54.8 v .1 .5 \%, P<0.01)$ and require more hysterectomies $(26.2$ v. $0.04 \%, P<0.01)^{(2)}$. Newborn infants delivered after uterine rupture are more frequently given Apgar scores lower than 5 at $5 \mathrm{~min}$, and have higher rates of perinatal mortality than those delivered without rupture ${ }^{(2)}$. Hence, it is critical to avoid uterine rupture. As evidenced by previous studies, women who had a caesarean section during previous pregnancy had an almost tenfold greater risk of uterine rupture than the women in the intact uterus group $(0.3 v .0 .03 \%)^{(3)}$, and women with a history of two prior caesarean deliveries had an almost fivefold greater risk than those with only one prior caesarean delivery ${ }^{(4)}$. Therefore, we assume that the healing of the uterine wound can influence the risk of rupture, and that a sound healing of the uterus can decrease the risk of rupture.

Nutrition plays an important role in the wound healing process $^{(5)}$; the major physiological changes, such as collagen synthesis, angiogenesis and wound contraction, need both energy and nutritional substrates. In the present study, we focused on whey peptides (WP), which are bioactive peptides derived from whey proteins. Based on previous studies, as well as on large-scale amino acid composition, we believe that WP might help the healing process of a caesarean section wound. Firstly, whey proteins are beneficial for experimental burn injury recovery in terms of decreasing oedema and inflammation, suppressing hepatic and renal oxidative stress measurements $^{(6)}$, and increasing glutathione synthesis and cellular antioxidant defence after experimental laparotomy and colonic anastomosis ${ }^{(7)}$. Secondly, compared with whey proteins, WP have a low molecular weight, and are therefore easier to digest and absorb ${ }^{(8)}$. Thirdly, many wound healing-related functions of WP have been found, such as antioxidation, immunomodulation and promotion of ileal mucosa, villus height and gastric secretion ${ }^{(9,10)}$. The purpose of the present study was to investigate the wound healing potential of WP in postcaesarean rats by biomechanical, biochemical and histological analyses.

\section{Materials and methods}

\section{Whey peptides}

WP, derivatives of whey proteins, were donated by the China CF Haishi Biotechnology Company Limited (Beijing, China). Firstly, whey proteins were homogenised and emulsified in distilled water. Secondly, at $40^{\circ} \mathrm{C}$ and at a $\mathrm{pH}$ of 8 , a complex protease was added $(0.03 \mathrm{~g} / \mathrm{g}$ protein) for $3 \mathrm{~h}$ before inactivation and sterile filtration. Finally, the mixture was made into a final powder by spray drying. The composition of the amino acids of the WP was determined (Table 1) using an amino acid analyser (835-50 Hitachi; Tokyo, Japan).

Abbreviations: bw, body weight; Hyp, hydroxyproline; WP, whey peptides.

$\dagger$ Both authors contributed equally to this work.

* Corresponding author: Professor Y. Li, fax +8610 82801177, email liyongbmu@163.com 
Table 1. Composition of the whey peptides ( $g / 100 \mathrm{~g})$

\begin{tabular}{lrll}
\hline Glu & 12.13 & Phe & 2.60 \\
Leu & 8.13 & Tyr & 2.45 \\
Asp & 7.84 & Arg & 1.86 \\
Lys & 6.63 & Met & 1.63 \\
Thr & 5.13 & Gly & 1.44 \\
Val & 4.35 & $\mathrm{NH}_{3}$ & 1.35 \\
Ile & 4.32 & His & 1.29 \\
Pro & 4.07 & Trp & 1.00 \\
Ala & 3.81 & Cys & 0.53 \\
Ser & 3.50 & & \\
Total & 74.06 & & \\
\hline
\end{tabular}

\section{Caesarean section model}

The institutional and national guidelines for the care and use of animals were followed, and all the experimental procedures involving animals were approved by the Animal Ethics Review Committee of Peking University Health Science Center. Forty-eight pregnant Sprague-Dawley rats were obtained from the Animal Service of Peking University, Health Science Center at $14 \mathrm{~d}$ of gestation. They were housed individually with free access to tap water and standard rat chow in an approved animal facility. A time period of $5 \mathrm{~d}$ was allowed for them to become acclimatised to the new environment. On day 19 (0.9 of gestation) of pregnancy, the rats were anaesthetised using intraperitoneal sodium pentobarbital $(40 \mathrm{mg} / \mathrm{kg})$. The caesarean section was based on Bowers's procedure ${ }^{(11)}$. A $3.0 \mathrm{~cm}$ midline laparotomy incision was made in the lower abdomen, and then a $2.0 \mathrm{~cm}$ longitudinal incision was made along the antimesenteric border in the midportion of each uterine horn. The rat pups and placentas were gently extruded through the hysterotomy. Afterwards, the uterine incisions were closed with a continuous nonlocking 5-0 polyglycolic acid suture, and the deep abdominal fascia as well as peritoneum was closed with 4-0 polyglycolic acid sutures. The skin was closed separately using the same material.

The rats were randomly assigned to two groups: control group and $1.13 \mathrm{~g} / \mathrm{kg}$ body weight (bw) WP group. The control group was given the AIN-93G diet, which is recommended to support growth, pregnancy and lactation by the American Institute of Nutrition ${ }^{(12)}$. WP and control vehicle were intragastrically administered every morning since the surgery day. In order to determine the effects of WP on bw and to adjust the injection volumes, we weighed the rats weekly. Meanwhile, the amount of chow eaten by each rat was determined to calculate the energy and protein intakes per week in the first 2 weeks. On days 7,14 and 21 after the surgery, eight rats of each group were randomly selected, anaesthetised by $\mathrm{CO}_{2}$ inhalation and killed by cervical dislocation. The blood samples were collected and used to determine serum total protein and albumin concentrations (Hitachi 7180 fully automatic biochemical analyser). The skin wounds and adjoining normal skin were harvested and separated into three equal strips with the wound at midline, which were then used for the tensile strength test, hydroxyproline (Hyp) and histological analyses, respectively. The adhesion in the abdominal cavity was scored based on the length and strength of macroscopic fibrous adhesion, which is a modified method from previous papers ( 0 , no adhesion; 1 , mild; 2, moderate; 3 , severe;

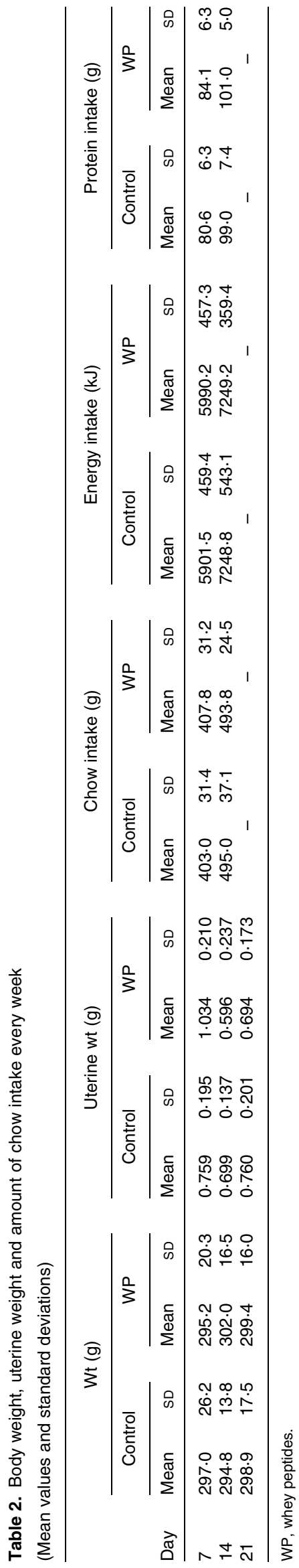


and 4, more severe $)^{(13)}$. The uterus was removed and weighed. The left uterine horn was used to measure the bursting pressure, while the right uterine horn was used for Hyp and histological analyses; four samples were used for each analysis.

\section{Measurement of tensile strength and bursting pressure}

The tensile strength and bursting pressure were measured using a pressure transducer and an amplifier-recorder (Biomedical Signal Acquiring Processing Systems; Beijing MicroStar Technology Development Company Limited, Beijing, China).

The $5 \times 10 \mathrm{~mm}^{2}$ skin strip was gripped at the wound edge and pulled slowly in the opposite direction until breakage. Tensile strength was calculated by dividing the breaking strength by the cross-sectional area ${ }^{(14)}$.

The uterine horns were suture ligated $1.0 \mathrm{~cm}$ distal to the uterine scar to prevent the leakage of perfusion fluid from the fallopian tubes. The horn was opened transversely $1 \mathrm{~cm}$ proximal to the scar. A catheter was inserted into the lumen and fixed in place with a suture. The proximal end of the catheter was connected to a $5 \mathrm{ml}$ injector, and a pressure transducer was connected by a three-way valve. The pressure transducer was connected to an amplifier-recorder. Normal saline was infused into each horn slowly until the horn burst and leakage occurred. The highest pressure that was obtained was designated the bursting pressure ${ }^{(11)}$.

\section{Quantitative analysis of hydroxyproline}

The skin and uterine samples collected for Hyp content assay were trimmed into rectangular pieces, which were about $20 \mathrm{mg}$. Hyp concentration was measured by a chemical colorimetric method using a commercial detection kit (A030 Hydroxyproline Detection Kit; Nanjing Jiancheng Bioengineering Institute, Nanjing, China).

\section{Histological analysis}

The skin and uterine specimens were collected vertically from the longitudinal scar. Morphological changes, including cellular content (neutrophils, macrophages and fibroblasts), collagen regeneration and vascularisation were detected by haematoxylin and eosin staining, as described in a previous paper $^{(15)}$. Masson's trichrome staining was used to evaluate the scar width in the skin and the degree of collagen formation due to its ability to stain collagen to a blue colour $^{(16)}$. Morphological findings, including cellular content (neutrophils, macrophages and fibroblasts), collagen formation and vascularisation, were scored as follows: 'none', - ; 'few', + ; 'moderate', ++ ; and 'many', $+++{ }^{(15)}$. Two independent pathologists performed the histological examination, and applied the scoring system in a blinded fashion.

\section{Statistical analysis}

Data were expressed as means and standard deviations, and statistical significance between the experimental and control values was analysed using Student's $t$ test, except the scores of histological findings, which were analysed using the Mann-Whitney $U$ test. The software SPSS 16.0 (SPSS, Inc., Chicago, IL, USA) was used, and a $P$-value $<0.05$ was considered statistically significant.

\section{Results}

The bw, uterine weight and chow intake in the two groups did not show a significant difference on days 7, 14 and 21 (Table 2). The individual data of chow intake on day 21 were unavailable because the rats were housed in groups after day 14 when the wound in the skin was well healed. Based on the chow intake and peptides administered by gavage, the energy and protein intakes were calculated and compared. They did not differ significantly between the groups (Table 2). More importantly, the adhesion levels of the uterine incision in the fatty tissue in the abdominal cavity were also similar.

\section{Total protein and albumin concentrations in serum}

The total protein concentration in serum did not increase, while the albumin concentration increased significantly on day $21(P=0.033$, Table 3$)$ due to excess peptide intake. The ratio of albumin:globulin in the WP group (2.4 (SD $0 \cdot 3)$ ) was significantly high than that of albumin:globulin in the control group $(2 \cdot 0(\operatorname{SD~} 0 \cdot 2))$ on day $7(P=0.032$, Table 3$)$.

\section{Tensile strength and bursting pressure}

The skin tensile strength and uterine bursting pressure kept increasing from day 7 to day 21. The WP group had greater skin tensile strength at all time points with a significant increase than the control group on day 7 (103.7 (SD 33.7)

Table 3. Total protein (TP) and albumin (Alb) concentration in serum after surgery (Mean values and standard deviations)

\begin{tabular}{|c|c|c|c|c|c|c|c|c|c|c|c|c|}
\hline \multirow[b]{3}{*}{ Day } & \multicolumn{4}{|c|}{ TP } & \multicolumn{4}{|c|}{ Alb } & \multicolumn{4}{|c|}{$A: G$} \\
\hline & \multicolumn{2}{|c|}{ Control } & \multicolumn{2}{|c|}{ WP } & \multicolumn{2}{|c|}{ Control } & \multicolumn{2}{|c|}{ WP } & \multicolumn{2}{|c|}{ Control } & \multicolumn{2}{|c|}{ WP } \\
\hline & Mean & SD & Mean & SD & Mean & $\mathrm{SD}$ & Mean & $\mathrm{SD}$ & Mean & SD & Mean & SD \\
\hline 7 & 71.2 & 5.6 & 64.5 & 8.7 & $47 \cdot 7$ & $2 \cdot 8$ & $45 \cdot 2$ & 5.0 & 2.0 & 0.2 & $2 \cdot 4^{*}$ & 0.3 \\
\hline 14 & 64.8 & 11.5 & 60.0 & $6 \cdot 0$ & $46 \cdot 8$ & $6 \cdot 8$ & 43.0 & $6 \cdot 2$ & $2 \cdot 7$ & 0.4 & $2 \cdot 6$ & 0.5 \\
\hline 21 & 59.9 & 9.9 & 71.9 & $10 \cdot 3$ & $42 \cdot 0$ & 5.4 & $49 \cdot 7^{\star}$ & 5.9 & $2 \cdot 5$ & 0.4 & $2 \cdot 3$ & 0.3 \\
\hline
\end{tabular}

A:G, albumin:globulin; WP, whey peptides.

* Mean value was significantly different from that of the control group $(P<0.05)$. 
Table 4. Skin tensile strength and uterine bursting strength after surgery (Mean values and standard deviations)

\begin{tabular}{|c|c|c|c|c|c|c|c|c|}
\hline \multirow[b]{3}{*}{ Day } & \multicolumn{4}{|c|}{ Skin tensile strength $\left(\mathrm{g} / \mathrm{mm}^{2}\right)$} & \multicolumn{4}{|c|}{ Uterine bursting strength $(\mathrm{mmHg})$} \\
\hline & \multicolumn{2}{|c|}{ Control } & \multicolumn{2}{|c|}{ WP } & \multicolumn{2}{|c|}{ Control } & \multicolumn{2}{|c|}{ WP } \\
\hline & Mean & SD & Mean & SD & Mean & SD & Mean & SD \\
\hline 7 & $40 \cdot 0$ & $12 \cdot 4$ & $103 \cdot 7^{\star}$ & 33.7 & $421 \cdot 7$ & 75.4 & $514 \cdot 1^{\star}$ & 61.4 \\
\hline 14 & 97.6 & $40 \cdot 0$ & $128 \cdot 3$ & $41 \cdot 8$ & 431.4 & 83.3 & $521 \cdot 8$ & 91.7 \\
\hline 21 & $150 \cdot 7$ & 65.7 & $162 \cdot 1$ & 37.7 & 580.4 & 63.1 & 629.8 & 92.2 \\
\hline
\end{tabular}

WP, whey peptides.

* Mean value was significantly different from that of the control group $(P<0.05)$.

v. 40.0 (SD 12.4) $\mathrm{g} / \mathrm{mm}^{2}$, Table 4). Similarly, the uterine bursting pressure in the WP group showed a trend to increase, with a significant difference on day 7 and a marginal significant difference on day $14(P=0.048$ and 0.068 , respectively; Table 4).

\section{Quantitative analysis of hydroxyproline}

Collagen deposition plays an important role in granulation tissue formation, and Hyp level indicates the amount of collagen. In the skin, the Hyp concentration increased significantly in the WP group on days 14 and 21 (Fig. 1(a)), while in the uterus, the increase in Hyp concentration did not show a significant difference on all three observation days (Fig. 1(b)).

\section{Histological analysis}

Skin tissue. Both the groups showed enhanced fibroblast and macrophage migration, vascularisation and collagen regeneration $7 \mathrm{~d}$ after the surgery. However, the neutrophil count in the WP group was lower than that in the control group $(P=0.032)$. The scar width was smaller (308 (SD 38) v. 408 (SD 64) $\mu \mathrm{m}, P=0.036$ ) and the collagen fibre was better organised in the WP group (Fig. 2(a)-(f), the gap between the two black arrowheads is the scar width in each section). The inflammatory cell infiltration and the scar width decreased and collagen fibre regeneration increased in both the groups $14 \mathrm{~d}$ after the surgery (Fig. 2(c) and (d)). The scar could be hardly identified, and cellular contents were similar in the unwounded tissue $21 \mathrm{~d}$ after the surgery (Fig. 2(e) and (f)).

Uterine tissue. The pattern of change in the cellular count was similar to that observed in the skin wound: a mass of inflammatory cells infiltrated to the wound site on day 7, and then the infiltration decreased. An increased density of blood vessels and collagen fibre was also detected in the regions of the lesions. However, there was no significant difference between the groups.

\section{Discussion}

In the present study, the healing process following a caesarean section in rats was studied. Wound healing is a complicated process, which is often artificially compartmentalised into three phases: inflammation, proliferation and maturation ${ }^{(17)}$. However, all the phases overlap to a certain degree, and none of these phases corresponds to a precisely defined period of time. The healing of the skin has been studied for many years, and it is well understood. The three time periods of observation that we chose, 7th day, 14th day and 21st day after incision, reflect the end of inflammation, proliferation and maturation. We did not conduct any observation during the first few days of incision, namely, the first $3 \mathrm{~d}$, because we thought that the WP may not have an acute effect. Also, we stopped the observation on the 21st day because from our previous experience we understood that the skin wound would be fully recovered from its appearance at this time point.
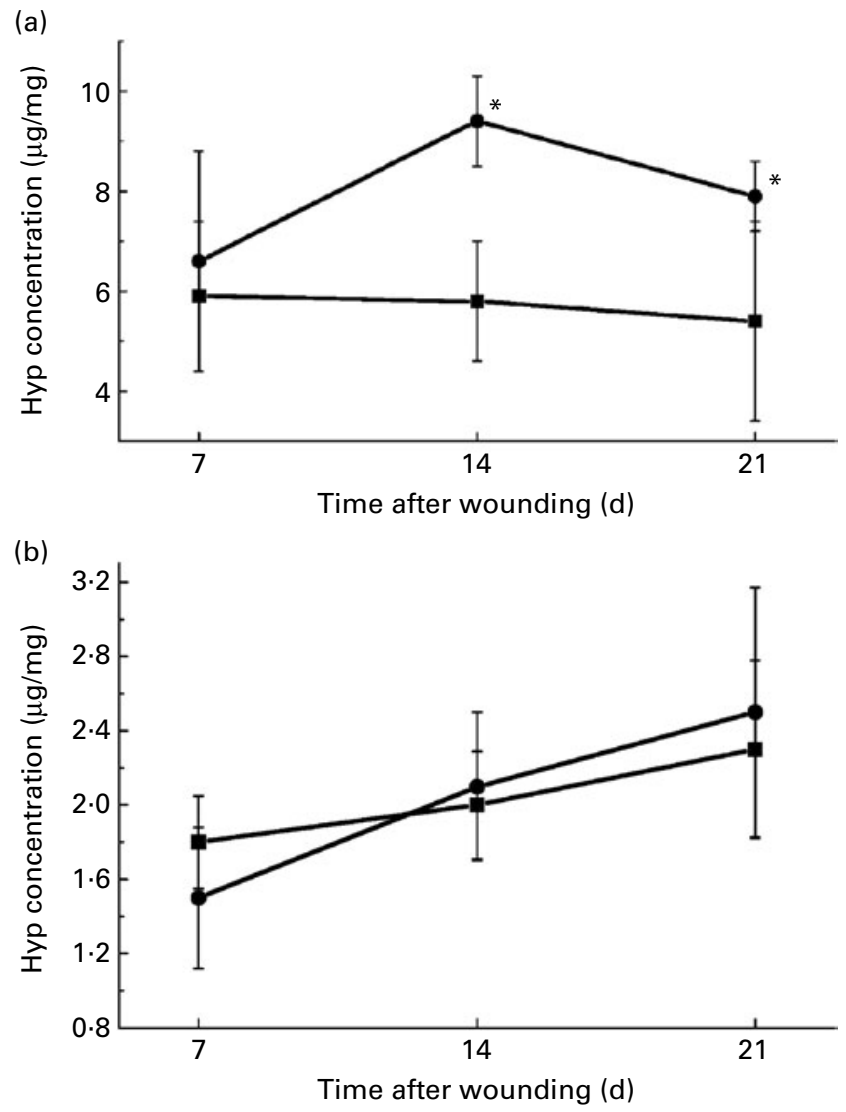

Fig. 1. Changes in the hydroxyproline (Hyp) concentration with time in the (a) skin and (b) uterus between the whey peptides (WP) group (•) and control group ( $\square$ ) on days 7,14 and 21 after the surgery. ${ }^{*}$ Mean value was significantly different from that of the control group $(P<0.05)$. 

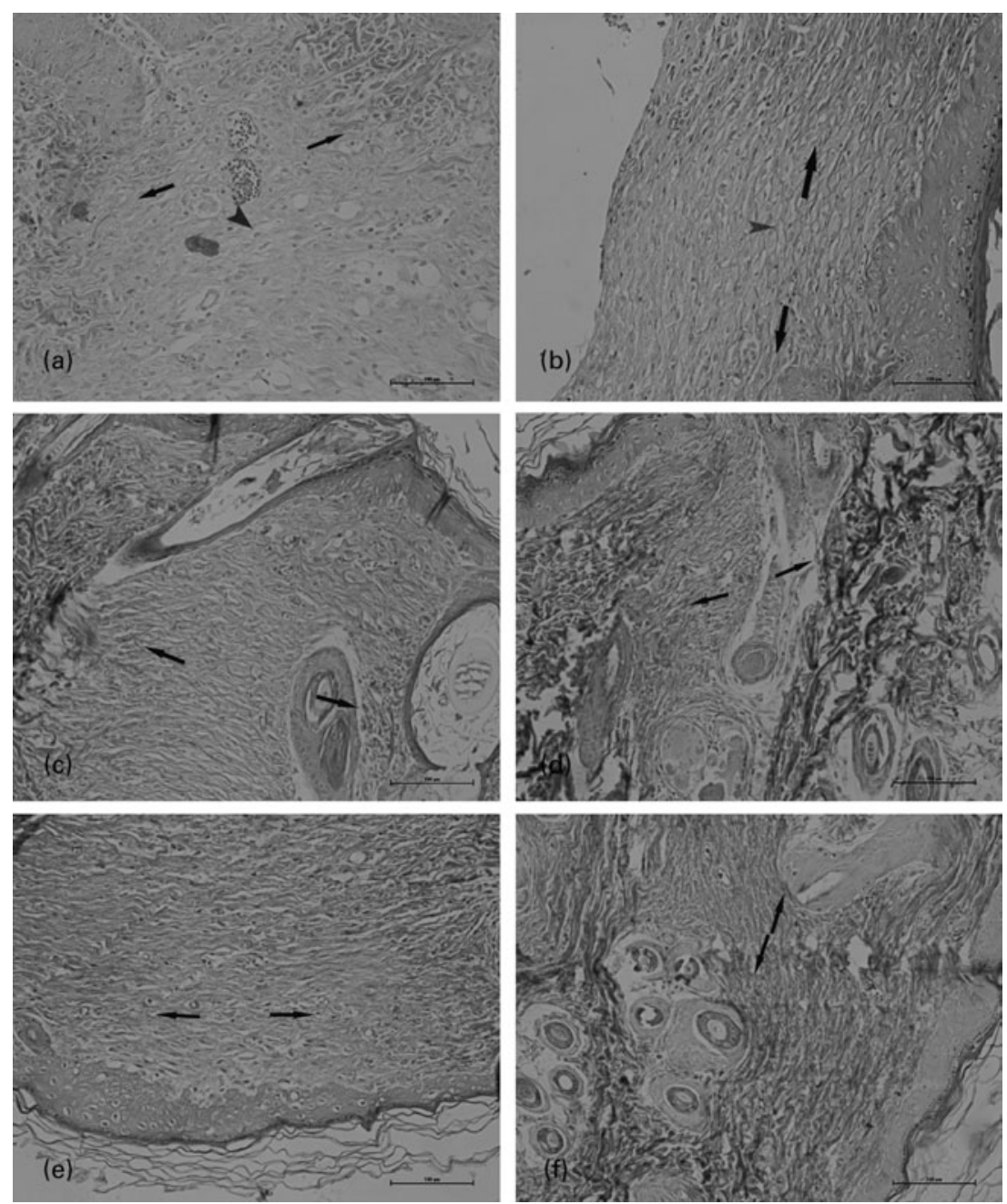

Fig. 2. Masson's staining of the skin incision on day 7 ((a) and (b)), day $14((\mathrm{c})$ and (d)) and day $21((\mathrm{e})$ and (f)). ((b), (d) and (f)) The staining patterns observed in the whey peptides (WP) group. The gap between the paired arrowheads is the scar width in each section. On day 21 , the scar could be hardly identified. The collagen fibres were more and better organised in the WP group (single arrowhead in (b)) than in the control group (single arrowhead in (a)) on day 7.

The present study measured breaking strength, Hyp concentration and histological property of the skin and uterus to evaluate the healing process after a caesarean section. Our data demonstrated that dietary WP increase the tensile strength and Hyp concentration, and decrease the scar width.

The tensile strength is the most common indicator in the evaluation of wound healing, both in the skin and in the uterus $^{(11,14)}$. As the tensile strength of the scarred tissue is much less than that of the normal tissue, a higher tensile strength reflects a faster healing process. The uterine scar tensile strength is always reflected by the uterine bursting pressure. Bower's study on rats ${ }^{(11)}$ showed that the increase in bursting pressure after the first caesarean section paralleled the increase in the second pregnancy. In the present study, we found that dietary WP increased the tensile strength of the skin and bursting pressure of the uterus in postcaesarean rats, which may predict faster healing and a decrease in the risk of uterine rupture in the WP group.

In the present study, the Hyp concentration changed differently in the skin and uterus. WP treatment increased Hyp concentration in the skin incision, but not that in the uterine incision. In the skin, Hyp concentration, as an indicator of collagen deposition, always has a positive correlation with tensile strength ${ }^{(18)}$, which is similar to the result obtained in the present study. However, previous studies held different ideas on the meaning of collagen content in uterine tissues ${ }^{(11,19,20)}$. Therefore, the finding that Hyp concentrations in the uterus were similar in the two groups in the present study indicates neither more nor less possibilities of uterine rupture. In the case of uterine dehiscence, Pollio et al. ${ }^{(21)}$ found that the scarred uterine segment showed a marked decrease or absence of transforming growth factor- $\beta 3$, a reduction in connective tissue growth factor concentration and an increase in basic fibroblast growth factor concentration. However, this result was obtained from a study conducted on human subjects, and we did not find any research on the growth factors in rat uteri. We do not know if they have the same trend yet. So, more basic research is necessary before drawing a clear conclusion.

In the present study, we administered WP to rats. In a paper on the antihypertensive effects of WP, a dose of about $3 \mathrm{~g} / \mathrm{kg}$ bw was used ${ }^{(22)}$. Based on this dose, we set a series 
of dosages that ranged from 0.4 to $3.4 \mathrm{~g} / \mathrm{kg}$. After killing the rats, tensile strength and bursting pressure were determined. The $1.13 \mathrm{~g} / \mathrm{kg}$ WP group was the group that showed a significant increase (data not shown). Further research was done on the $1.13 \mathrm{~g} / \mathrm{kg}$ bw WP group and the control group. In the hypermetabolic state, postpartum or postsurgery period, for instance, the body needs a higher amino acid intake to guarantee collagen production. Although the intakes of the total amount of amino acids that were calculated were similar between the groups, as the peptides are believed to be absorbed more easily than proteins ${ }^{(8)}$, we tested if the absorbance would affect the proteins in the body, which may also influence wound healing. Serum albumin and total protein were chosen to reflect the proteins in the body. The increase in serum albumin concentration on day 21 indicated such a possibility, which may partly explain the increase in skin Hyp concentration on days 14 and 21 after the surgery. However, albumin has a long half-life of about $20 \mathrm{~d}$, which may cause a delayed increase in the serum albumin concentration until day 21. In future studies, we may use other faster parameters, such as prealbumin, to evaluate the protein level in the body. Also, the abundant amount of glutamic acid in WP, which may be transferred to glutamine in the body, can contribute to the promotion of healing effect, since glutamine is a fuel source for rapidly dividing cells such as fibroblasts, epithelial cells, lymphocytes and macrophages ${ }^{(5)}$.

The $\mathrm{T}$ lymphocytes play an important role in the healing process. Previous study has revealed that suppressed cellular immunity delayed open wound contraction ${ }^{(23)}$. However, a successful pregnancy needs a down-regulation of the cellular immune response in order not to reject the fetus ${ }^{(24)}$. This may hinder the healing after delivery. Previous research has found that some of the WP significantly enhanced the proliferation of peripheral blood lymphocytes ${ }^{(25)}$. Therefore, the supplementation of WP after a caesarean section may promote wound healing by the up-regulation of the cellular immune response.

Different from the lymphocytes, a decrease in the neutrophil infiltration in the section is preferred. Neutrophils release a large amount of highly active reactive oxygen species in the wound ${ }^{(26)}$, which results in oxidative stress and causes excessive tissue loss, thereby causing excessive scar formation and delayed wound healing ${ }^{(16,27)}$. It has been demonstrated that neutrophil-depleted mice exhibit faster wound closure and less scar than the control mice ${ }^{(16,28)}$. The present study found a low neutrophil count in the WP group, which may explain the decrease in scar width.

Dietary whey supplementation can also increase glutathione synthesis in wounded rats ${ }^{(7)}$. The levels of glutathione decreased by $60-70 \%$ in the acute wounds compared with in the normal skin ${ }^{(29)}$. The levels of glutathione in the wound tissue are positively related with wound bursting strength $^{(30)}$ not only because it is an antioxidant ${ }^{(29)}$, but also because it can increase the $\mathrm{CD}^{+}$cells: $\mathrm{CD}^{+}$cells ratio ${ }^{(31)}$. Both these reasons can be attributed to the stronger tensile strength and better collagenation that were observed in the present study ${ }^{(32)}$

In the present study, WP had a clearer effect on the promotion of healing in skin than in the uterus. In the uterus, except for bursting strength, no significant increase was found in the WP group. We checked the PubMed database for the papers on uterine healing in the last 20 years, but we only found a few animal research papers. The lack of relative data caused a difficulty in conducting research exploring a clear conclusion in rats any further. Currently, more results on clinical observations that provide more indicators to evaluate uterine healing are being found. Moreover, compared with rat uterus, human uterus has a different anatomical structure, which leads to more adhesion to the peritoneum. Because of this, we suggest that further study be conducted on human subjects.

In conclusion, the present study indicated that WP might promote recovery of wounds in rats after a caesarean section. The reason for WP possessing significant wound healing potential may be due to their multitarget therapy properties, which have made them a new treatment concept for wounds ${ }^{(33)}$. This kind of natural source for nutritional intervention may be beneficial for the puerpera. However, more studies are needed to ensure the functions, and explore the mechanisms of the WP in wound healing process.

\section{Acknowledgements}

This work was supported by the foundation (no. 2006BAD27B01) from the Ministry of Science and Technology of the People's Republic of China. There is no conflict of interest to be disclosed. Y. L. gave the original idea, and was in charge of the whole trial. J. W. and M. Z. conducted the animal experiment and wrote the whole paper. R. L., Z. Z., H. Z., J. Z. and T. L. assisted in the animal trials and chemical analyses.

\section{References}

1. Hamilton BE, Martin JA \& Ventura SJ (2006) Births: preliminary data for 2005. Natl Vital Stat Rep 55, 1-18.

2. Ofir K, Sheiner E, Levy A, et al. (2003) Uterine rupture: risk factors and pregnancy outcome. Am J Obstet Gynecol 189, $1042-1046$.

3. Locatelli A, Ghidini A, Ciriello E, et al. (2006) Induction of labor: comparison of a cohort with uterine scar from previous cesarean section vs. a cohort with intact uterus. J Matern Fetal Neonatal Med 19, 471-475.

4. Caughey AB, Shipp TD, Repke JT, et al. (1999) Rate of uterine rupture during a trial of labor in women with one or two prior cesarean deliveries. Am J Obstet Gynecol 181, 872-876.

5. Thompson C \& Fuhrman MP (2005) Nutrients and wound healing: still searching for the magic bullet. Nutr Clin Pract 20, $331-347$.

6. Oner OZ, Ogunc AV, Cingi A, et al. (2006) Whey feeding suppresses the measurement of oxidative stress in experimental burn injury. Surg Today 36, 376-381.

7. Ogunc AV, Manukyan M, Cingi A, et al. (2008) Dietary whey supplementation in experimental models of wound healing. Int $J$ Vitam Nutr Res 78, 70-73.

8. Meisel H (2007) Food-derived bioactive proteins and peptides as potential components of nutraceuticals. Curr Pharm Des 13, 873-874.

9. Meisel H (2005) Biochemical properties of peptides encrypted in bovine milk proteins. Curr Med Chem 12, 1905-1919.

10. Peng X, Xiong YL \& Kong B (2009) Antioxidant activity of peptide fractions from whey protein hydrolysates as measured by electron spin resonance. Food Chem 113, 196-201. 
11. Bowers D, McKenzie D, Dutta D, et al. (2001) Growth hormone treatment after cesarean delivery in rats increases the strength of the uterine scar. Am J Obstet Gynecol 185, 614-617.

12. Reeves PG, Nielsen FH \& Fahey GJ (1993) AIN-93 purified diets for laboratory rodents: final report of the American Institute of Nutrition ad hoc writing committee on the reformulation of the AIN-76A rodent diet. J Nutr 123, 1939-1951.

13. Gul A, Kotan C, Ugras S, et al. (2001) Transverse uterine incision non-closure versus closure: an experimental study in dogs. Eur J Obstet Gynecol Reprod Biol 88, 95-99.

14. Eroglu E, Eroglu F, Agalar F, et al. (2001) The effect of lidocaine/prilocaine cream on an experimental wound healing model. Eur J Emerg Med 8, 199-201.

15. Qiu Z, Kwon AH \& Kamiyama Y (2007) Effects of plasma fibronectin on the healing of full-thickness skin wounds in streptozotocin-induced diabetic rats. J Surg Res 138, 64-70.

16. Wilgus TA, Vodovotz Y, Vittadini E, et al. (2003) Reduction of scar formation in full-thickness wounds with topical celecoxib treatment. Wound Repair Regen 11, 25-34.

17. Baum CL \& Arpey CJ (2005) Normal cutaneous wound healing: clinical correlation with cellular and molecular events. Dermatol Surg 31, 674-686.

18. Perez GR, Vargas SR \& Ortiz HY (2005) Wound healing properties of Hylocereus undatus on diabetic rats. Phytother Res 19, 665-668.

19. Pettersson KW, Grunewald C \& Thomassen P (2007) Uterine rupture and perinatal outcome. Acta Obstet Gyn Scan 86, 1337-1341.

20. Buhimschi CS, Buhimschi IA, Yu CL, et al. (2006) The effect of dystocia and previous cesarean uterine scar on the tensile properties of the lower uterine segment. Am J Obstet Gynecol 194, 873-883.

21. Pollio F, Staibano S, Mascolo M, et al. (2006) Uterine dehiscence in term pregnant patients with one previous cesarean delivery: growth factor immunoexpression and collagen content in the scarred lower uterine segment. Am J Obstet Gynecol 194, $527-534$
22. Tsai JS, Chen TJ, Pan BS, et al. (2008) Antihypertensive effect of bioactive peptides produced by protease-facilitated lactic acid fermentation of milk. Food Chem 106, 552-558.

23. Feeser VR, Menke NB, Ward KR, et al. (2009) Androstenediol reverses steroid-inhibited wound healing. Wound Repair Regen 17, 758-761.

24. Reinhard G, Noll A, Schlebusch H, et al. (1998) Shifts in the $\mathrm{TH} 1 / \mathrm{TH} 2$ balance during human pregnancy correlate with apoptotic changes. Biochem Biophys Res Commun 245, 933-938.

25. Kayser H \& Meisel H (1996) Stimulation of human peripheral blood lymphocytes by bioactive peptides derived from bovine milk proteins. FEBS Lett 383, 18-20.

26. Eming SA, Hammerschmidt M, Krieg T, et al. (2009) Interrelation of immunity and tissue repair or regeneration. Semin Cell Dev Biol 20, 517-527.

27. Dissemond J, Goos M \& Wagner SN (2002) The role of oxidative stress in the pathogenesis and therapy of chronic wounds. Hautarzt 53, 718-723.

28. Dovi JV, He LK \& DiPietro LA (2003) Accelerated wound closure in neutrophil-depleted mice. J Leukoc Biol 73, 448-455.

29. Shukla A, Rasik AM \& Patnaik GK (1997) Depletion of reduced glutathione, ascorbic acid, vitamin $\mathrm{E}$ and antioxidant defence enzymes in a healing cutaneous wound. Free Radic Res 26, 93-101.

30. Adamson B, Schwarz D, Klugston P, et al. (1996) Delayed repair: the role of glutathione in a rat incisional wound model. J Surg Res 62, 159-164.

31. Pathak $\mathrm{N} \&$ Khandelwal $\mathrm{S}$ (2008) Impact of cadmium in $\mathrm{T}$ lymphocyte subsets and cytokine expression: differential regulation by oxidative stress and apoptosis. Biometals 21, 179-187.

32. Shetty S, Udupa S \& Udupa L (2008) Evaluation of antioxidant and wound healing effects of alcoholic and aqueous extract of Ocimum sanctum Linn in rats. Evid Based Complement Alternat Med 5, 95-101.

33. Gupta A, Upadhyay NK, Sawhney RC, et al. (2008) A poly-herbal formulation accelerates normal and impaired diabetic wound healing. Wound Repair Regen 16, 784-790. 